\title{
Percepção social e cognição em situações de aprendizagem por conflito sociocognitivo
}

\author{
Fernanda Dias
}

\begin{abstract}
Resumo
O objetivo desta pesquisa foi investigar as relações entre a aprendizagem mediadas pelo conflito sociocognitivo e o grau de percepção dos sujeitos sobre sua posição sociométrica no grupo. Os sujeitos da pesquisa foram crianças de 5 a 7 anos; os procedimentos metodológicos consistiram de pré-teste, três sessões de intervenção e pós-teste. Foram aplicadas provas operatória e de possíveis, teste sociométrico e perceptual. Os resultados mostraram efeitos positivos da intervenção por conflito sociocognitivo. Com base nos dados para conservação de comprimento pôdese afirmar apenas uma tendência para a relação melhor percepção/maior aprendizagem, não estatisticamente significativa. Quanto à situação de aprendizagem para formas de uma realidade parcialmente escondida, cada uma das situações sociométricas isoladamente não pôde explicar a aprendizagem; entretanto, ao considerar-se a percepção como um todo, a relação maior-percepção/melhor-aprendizagem pôde ser afirmada.

Palavras-Chave: Percepção social; Aprendizagem; Conflito sociocognitivo.
\end{abstract}

\section{Social perception and cognition in learning situations through social-cognitive conflict}

\begin{abstract}
The objective of this research was to investigate the relationship between the learning mediated by social-cognitive conflict and the degree of individuals' perception of their own sociometric status in the group. The subjects are children between from 5 to 7 years old; the experimental procedures consisted of pre-tests, three intervention sessions and post-test. Operative and formation of possibilities tasks as well as sociometric and perceptual tests were applied. The results showed positive effects from the intervention of social-cognitive conflict. Based on the data of length conservation learning, only a slight tendency for the relation better level of perception, higher level of learning could be confirmed but it was not statistically significant. In relation to the learning of forms of partially hidden reality, each separated sociometric situation could not explain the learning, but considering the perception as a whole, the relation better perception, higher learning could be confirmed.
\end{abstract}

Keywords: Social perception; Learning; Social-cognitive conflict.

\section{Introdução}

Este estudo se insere no campo das investigações sobre as relações sociais, circunscrito às situações de aprendizagem, mediadas pelo conflito sociocognitivo. Fundamenta-se nos pressupostos da teoria piagetiana quanto aos conceitos de interação social e aprendizagem por conflito cognitivo (Sisto, 1993). Busca subsídios na teoria moreniana ao enfocar as relações interpessoais do ponto de vista da Sociometria, elegendo dessa teoria o teste sociométrico e o teste perceptual como instrumentos de estudo.

Encontram-se desde as primeiras obras de Piaget a valorização do fator social como necessário para a socialização progressiva do pensamento ao tratar de temas como egocentrismo, cooperação e descentração, afirmando o valor da interação social como essencial para o desenvolvimento cognitivo (Piaget, 1926, 1973, por exemplo). $\mathrm{Na}$ conduta humana distingue duas espécies de interações indissociáveis que a modificam e transformam as estruturas mentais do

Endereço para correspondência:

R. Osaka, 33, apto. 24, bloco 1 - Jd. Cláudia - Londrina-PR

E-mail: fe21@hotmail.com sujeito, a interação entre o sujeito e os objetos e a interação entre o sujeito e os outros sujeitos. Estabelece um paralelo entre o desenvolvimento do indivíduo e qualidade dos intercâmbios que mantém com o meio social, qualidade essa que transita desde um "egocentrismo" nas fases iniciais de desenvolvimento, passando pelas possibilidades de cooperação no período das operações concretas, até um pensamento propriamente coletivo na fase das operações formais.

Associado às pesquisas que enfatizam questões relativas à interação social e desenvolvimento cognitivo, um conceito emerge consistentemente nas pesquisas experimentais a partir da década de 70 , qual seja, a valorização do conflito cognitivo como fonte de progressos na aprendizagem (Sisto, 1993, 1997). A idéia de conflito cognitivo está associada à perturbação cognitiva na teoria de Piaget (1977) e se refere aos desequilíbrios provocados pelas perturbações cognitivas que se apresentam ao sujeito no seu enfrentamento com o ambiente. As reações do sujeito a essas perturbações procuram compensar as perturbações e geram novas construções. 
Há autores que reforçam a ênfase na dimensão social do conflito (Mugny, Doise \& Perret-Clermont, 1975/76) quando afirmam que as atividades cognitivas individuais adquirem significação nas interações sociais, reais ou simbólicas, que só serão estruturantes na medida em que suscitem um conflito de resposta entre os participantes. O conflito surge então como fonte de mudança, no indivíduo e nos sistemas sociais, tratandose, assim, de um conflito sociocognitivo.

As inúmeras pesquisas desenvolvidas nesse campo de investigação, quer no âmbito internacional (Mugny, Doise \& Giroud, 1975; Russel, 1982; Mugny \& Doise, 1983; Bearison, Magzamen \& Filardo, 1986; Emler \& Glachan,1988; Fraysse, 1991; Taal \& Oppenheimer, 1989; Tudge, 1989; Bjistra, Van Geert \& Jackson, 1989; Ganuza, 1996, entre outros) como nacional (Nunes, 1998; Troncoso Guerrero, 1998; Garcia, 1999; Cunha, 1999; para citar alguns), mostram o interesse crescente e os resultados positivos alcançados pela utilização do conflito sociocognitivo na situação experimental como meio de provocar aprendizagem. Nessas pesquisas encontraram-se subsídios quanto à estruturação das situações experimentais e quanto à natureza das tarefas propostas, elegendo-se a situação de interação social por conflito sociocognitivo para investigar o grau de percepção dos sujeitos sobre sua posição sociométrica no grupo e suas relações com situações de aprendizagem.

Para medida e análise de uma determinada estrutura social, Moreno (1972) cria o teste sociométrico, que determina a posição de cada indivíduo em um grupo no qual tenha uma função, por exemplo, no qual vive ou trabalha. Ao estudar o processo de escolha e rejeições no teste sociométrico, o fenômeno da percepção que cada indivíduo tem sobre sua posição sociométrica no grupo chama a atenção de Moreno e foi denominado de "auto-avaliação sociométrica". Surge assim o teste perceptual, que permite isolar o fator cognitivo nas escolhas e rejeições, evidenciando o nível em que o sujeito percebe de forma objetiva o que ocorre nas situações e o que se passa em relação às escolhas das outras pessoas. Pesquisas relatadas nesse campo (Tagiuri, 1952; Maisonneuve, 1970; Krantz, 1982; Priel \& Leshem, 1990; Patterson, Kupersmidt \& Griesler, 1990; entre outros), apontam para a validade das medidas sociométricas associadas ao seu complemento perceptivo sob a forma de testes e escalas, mesmo para crianças mais jovens, respaldando assim sua utilização no presente estudo.

Com base no embasamento propiciado pela teoria piagetiana e pela sociometria, o objetivo desta pesquisa foi investigar as relações entre maior permeabilidade do sistema cognitivo a situações de aprendizagem e o grau de percepção dos sujeitos sobre sua posição sociométrica no grupo, em situação de aprendizagem por conflito sociocognitivo de conservação de comprimento e em formas de uma realidade parcialmente escondida. A hipótese subjacente é a de que indivíduos com uma percepção social mais acurada de sua aceitabilidade ou rejeição em situação grupal estabelecerão relações mais adequadas com o meio e, conseqüentemente, com situações de aprendizagem, concebida como uma forma de adaptação ao ambiente.

\section{Método}

\section{Participantes}

Os sujeitos da pesquisa foram crianças com idade variando de 5 a 7 anos, de ambos os sexos. Para o experimento conservação de comprimento participaram 69 crianças e 36 crianças para o experimento formas possiveis de uma realidade parcialmente escondida. Todas as crianças freqüentavam pré-escola na rede municipal ou estadual de ensino; eram não conservadoras na prova de conservação e analógicas na prova de formação de possíveis. Foram compostos, por sorteio, o grupo experimental (GE) formado por duplas aleatórias de crianças, que passaram pelo procedimento experimental com conflito sociocognitivo, e o grupo de controle (GC) composto por crianças que não foram submetidas à intervenção de aprendizagem.

\section{Procedimentos}

Os procedimentos metodológicos incluíram pré-teste, intervenção e pós-teste, para ambos os experimentos, no caso do grupo experimental. Os gruposcontrole foram submetidos apenas aos pré e pós-testes.

\section{A-Pré e pós-testes}

No pré-teste os sujeitos foram submetidos às seguintes provas: Teste Sociométrico (Moreno, 1972) e Teste Perceptual (Bustos, 1979); uma prova operatória de conservação de comprimento (Piaget, Inhelder, Szeminska, 1948) e uma prova de formação de possíveis, formas possíveis de uma realidade parcialmente escondida (Piaget, 1985). A ordem de aplicação das provas foi aleatória, determinada por sorteio. Após a intervenção foram realizados os pós-testes, reaplicandose a prova operatória e a de formação de possíveis.

\section{$B$ - Intervenção}

A intervenção constou de duas sessões de modelos filmados e três sessões experimentais de aprendizagem por conflito (Sisto, 1993). Os sujeitos assistiram a um filme que mostrou quatro crianças realizando a prova de conservação de comprimento, exibindo condutas de conservação e não conservação. As três sessões experimentais, em situações de aprendizagem, foram realizadas em duplas, após a sessão de vídeo, constando de interação social com conflito sociocognitivo, com duração média de trinta minutos. Cada sessão consistiu 
de sete situações experimentais, nas quais foram introduzidas as situações de conflito (por contraposição às respostas dos sujeitos ou por contra-argumentos). $\mathrm{O}$ objetivo era levar a dupla a uma possível mudança de nível cognitivo.

\section{C - Aplicação dos testes e prova e avaliação.}

A administração dos testes Sociométrico e Perceptual seguiram as indicações de Moreno (1972) e Bustos (1979), utilizando-se como material papel e lápis, tendo sido elaborado um protocolo de registro para anotação das respostas dos sujeitos. Primeiramente foram solicitados três nomes de colegas de classe com quem gostaria de estudar e três nomes com quem gostaria de brincar; em seguida, três nomes com quem não gostaria de estudar e três nomes com quem não gostaria de brincar. Após essa parte, foi perguntado "quem você acha que escolheu você para estudar?" e "quem você acha que escolheu você para brincar?" e depois, quem ela acha que não a escolheu para as duas situações. O critério de avaliação seguiu as indicações de Bustos (1979) e para a pontuação foram consideradas as coincidências entre os sinais positivos (aceitação) e negativos (rejeição), para cada um dos critérios considerados (estudar e brincar).

Para a prova de conservação de comprimento foi realizada uma adaptação valendo-se da prova original (Piaget, Inhelder, Szeminska, 1948), com base em um estudo piloto. Os materiais utilizados foram palitos de madeira e uma folha de registro. A aplicação da prova seguiu o procedimento padrão proposto pelos autores, bem como sua avaliação, analisando-se três tipos de respostas quanto à conservação do comprimento: conservadores, quando apresentavam argumentos operatórios; intermediários, quando às vezes apresentavam argumentos operatórios e às vezes, não; e não-conservadores, quando não apresentavam argumentos operatórios.

Para a prova de formas possiveis de uma realidade parcialmente escondida foi realizada uma adaptação da prova original (Piaget, 1985), trabalhando-se com figuras geométricas coladas em pranchas e quadrados de papel sulfite utilizados para execução dos desenhos pelos sujeitos, contendo cada um o desenho de uma das figuras das pranchas. Foi utilizada caneta esferográfica para a execução dos desenhos. Os sujeitos foram classificados de acordo com os desenhos realizados para as três pranchas, em nível IA, quando os desenhos realizados fossem de um só tipo; IB, quando ocorriam variações; nível II, se ocorressem três ou mais variações e nível III, quando os sujeitos admitiam um número ilimitado ou infinito de variações.

\section{Resultados}

Os dados obtidos pelos sujeitos quanto à aprendiragem em comprimento no GE e resultados da intervenção evidenciam ganhos cognitivos ocorridos entre o pré-teste e o pós-I, bem como a estabilidade mantida entre os dois pós-testes. No GC a maioria dos sujeitos (89\% aproximadamente) não apresentou indícios de movimentação cognitiva.

A comparação entre os resultados dos GE e GC foi realizada confrontando-se os níveis de desempenho cognitivo alcançados e analisando-se as categorias de movimentos cognitivos (CAT, elaboradas por Sisto, 1997), em relação às freqüências observadas.

$\mathrm{Na}$ comparação entre os níveis cognitivos do GE e GC (Tabelas 1 e 2), os dados permitem concluir pela confirmação dos efeitos favoráveis para a aprendizagem, obtidos com a intervenção, não podendo as diferenças das médias obtidas ser atribuídas ao acaso $(\mathrm{t}=2,137$ e $\mathrm{p}=0,0336$ para pré-teste e pós-I e $\mathrm{t}=2,715 \mathrm{e}$ $\mathrm{p}=0,008$ para pré-teste e pós-II). Na segunda forma de análise os dados apontam claramente para uma maior movimentação cognitiva para o GE, embora nos dois grupos a categoria 10, ausência de movimento, seja elevada, tanto para o GE (63\%) como para GC $(89 \%)$.

Nas análises sobre percepsão e resultados da aprendizagem em comprimento, os dados obtidos foram estudados procurando-se verificar se a percepção para cada um dos critérios (estudar e brincar) e para a percepção em conjunto (perceptual geral) diferenciou os resultados do pós-teste I e pós-teste II. Os dados obtidos permitiram concluir que, considerando-se isoladamente os dois critérios, ou tomando-os em conjunto, não se pôde falar em uma relação estatisticamente significativa para aprendizagem e percepção. De fato, apenas o critério estudar evidenciou algum indício com vistas a se associar aos níveis perceptuais mais elevados com mudanças cognitivas, mas sem significação estatística.

Em resumo, os dados obtidos com base na análise da aprendizagem em comprimento evidenciaram a influência positiva da intervenção para promover ganhos cognitivos. Entretanto, quando se procurou relacionar os progressos cognitivos aos níveis de percepção, não foi confirmada essa possibilidade. Observou-se apenas uma tendência para a relação melhor percepção/maior aprendizagem, mas essa tendência não foi estatisticamente significativa.

Quanto à aprendizagem em realidade parcialmente escondida, o progresso cognitivo resultante da intervenção (GE) foi evidente e análise estatística realizada evidencia que as mudanças observadas não podem ser atribuídas ao acaso (pré-teste e pós-teste I, $\mathrm{t}=7,645$ e $\mathrm{p}=0,000)$. Entretanto, a análise da aprendizagem do GC mostrou que houve aprendizagens, mas as diferenças podem ser atribuídas ao acaso, tanto para os dados do pré-teste ao pós-I 
$(\mathrm{t}=1,835$ e $\mathrm{p}=0,104)$ como do pré-teste ao pós-II $(\mathrm{t}=2,294$ e $\mathrm{p}=0,051)$. A análise dos movimentos cognitivos por meio das categorias de aprendizagem mostrou para GE maior concentração de freqüências na categoria movimento positivo com estabilidade (40\%), enquanto para GC a maior concentração foi para ausência de movimento (55\%), o que indica a baixa mobilidade cognitiva para este grupo.

Para analisar-se conjuntamente os efeitos da percepção e resultados da aprendizagem em realidade parcialmente escondida e intervenção, observou-se a distribuição dos sujeitos quanto à aprendizagem do pré-teste para o pósI e do pré-teste para o pós-II e as categorias de aprendizagem (CAT) em relação ao grupo experimental, controle e tipo de intervenção, para cada uma das situações (estudar, brincar e perceptual geral).

A primeira análise permitiu afirmar que os sujeitos reagiram favoravelmente à intervenção e que houve aprendizagem, bem como uma tendência para a relação melhor percepção/maior aprendizagem. Entretanto, somente ao se juntarem as duas situações sociométricas, obtendo-se o perceptual geral, é que essa tendência mostrou-se estatisticamente significativa $(p=0,000$, tanto para o desempenho do pré-teste para o pós-testes I, quanto do pré-teste para pós-teste II).

Para a segunda análise, ligada à percepção considerada globalmente e suas relações com as categorias de aprendizagem, as diferenças entre os dois grupos (experimental e controle) não se mostraram significativas pela análise da variância $(\mathrm{p}=0,287 \mathrm{e} \mathrm{p}=$ $0,291)$, indicando que as diferenças encontradas podem ser casuais. A relação entre categorias de aprendizagem e perceptual geral ficou evidenciada pelo nível de significância alcançado $(p=0,00)$, mostrando que, ao tratar a percepção no conjunto, pôde-se afirmar a relação percepção/aprendizagem. No conjunto, os dados sugerem uma relação entre maior percepção e movimento positivo com estabilidade; quando relacionada a categorias de aprendizagem, a intervenção não apresenta efeitos $(p=0,57)$, pois toda variância está explicada pela percepção.

\section{Conclusões}

O objetivo deste trabalho foi o de investigar as possíveis relações entre a permeabilidade do sistema cognitivo às intervenções do meio em situações de aprendizagem e o grau de percepção dos sujeitos em suas relações interpessoais. Perguntou-se se crianças com um grau maior de percepção sobre sua posição sociométrica no grupo, portanto, com maior capacidade de adaptação ao meio, mostrariam essa plasticidade também em situações de aprendizagem.
Para responder a tais questões trabalhou-se com dois conteúdos diferentes em situações experimentais de aprendizagem (conservação de comprimento e formas de uma realidade parcialmente escondida) mediadas pelo conflito sociocognitivo, e buscaram-se dados sobre o grau de percepção dos sujeitos nas situações sociométricas de estudar e brincar, obtidos pela aplicação do teste perceptual, conforme Moreno (1972) e Bustos (1979).

Os efeitos da intervenção por conflito sociocognitivo mostraram-se positivos para as duas situações experimentais de aprendizagem, em consonância aos estudos de Sisto (1997); entretanto, observou-se, no caso da aprendizagem em comprimento, menor permeabilidade dos sujeitos para este conteúdo, expressada pela alta porcentagem de casos de ausência de movimentos cognitivos, tanto para o grupo-controle quanto para o grupo experimental. $O$ que poderia explicar essa maior permeabilidade do sistema cognitivo à intervenção pelo conteúdo relacionado aos possíveis?

Talvez uma das respostas possa estar ligada à natureza dos esquemas cognitivos envolvidos. A prova de comprimento solicitou predominantemente esquemas operatórios, exigindo um processo de auto-regulação de natureza normativa, um mecanismo dedutivo, mais laboriosamente construído pelo sistema cognitivo. Por sua vez, formas de uma realidade parcialmente escondida, ao exigirem esquemas de procedimento, apresentaram um caráter mais imediato e de contingência, de natureza criativa, oferecendo maior permeabilidade às situações de aprendizagem.

Um outro fato interessante constatado referiuse às duas situações sociométricas, estudar e brincar. Tanto na prova operatória como na dos possíveis, as crianças mostraram maiores dificuldades perceptivas para a situação estudar. Talvez o fato de ser essa a primeira situação sociométrica apresentada aos sujeitos tenha trazido maior impacto ao sistema cognitivo. Também não se pode desconsiderar que a situação brincar apresenta-se de forma mais próxima ao cotidiano de crianças pequenas, pré-escolares, como foi o caso da maioria dos sujeitos da pesquisa. Para essas crianças, brincar e estudar ainda são atividades bastante imbricadas e indiferenciadas, pois não se pode afirmar que haja situações nitidamente de estudo para a maioria das crianças pesquisadas, principalmente considerando-se que, na pré-escola freqüentada, as situações escolares são nítidas e predominantemente lúdicas.

Quanto ao grau de percepção dos sujeitos e a aprendizagem, observaram-se algumas particularidades para cada um dos conteúdos pesquisados. Para a situação de aprendizagem em comprimento não se observou relação entre percepção e ausência ou presença de movimentação cognitiva para nenhuma das situações 
sociométricas (estudar e brincar) e quando a percepção nas duas situações foi considerada globalmente. Entretanto, ao se relacionarem os níveis perceptuais a categorias de movimentos cognitivos, obtiveram-se, para o critério estudar indícios de associação entre níveis perceptuais e mudanças cognitivas.

No conjunto dos dados para comprimento, pôde-se afirmar apenas uma leve tendência para a relação melhor percepção/maior aprendizagem. No entanto, essa tendência não se mostrou estatisticamente significativa.

Quanto à situação de aprendizagem para formas de uma realidade parcialmente escondida, cada uma das situações sociométricas (estudar, brincar) isoladamente não pôde explicar a aprendizagem. Entretanto, juntando-se as duas situações (perceptual geral), a relação percepção/aprendizagem pôde ser afirmada. Nessa condição, a relação entre uma percepção mais acurada e ganhos cognitivos com estabilidade na aprendizagem foi observada, podendo-se falar de uma mudança significativa e não apenas sugerida.

Para explicar a diferença nos resultados das situações experimentais, uma possibilidade poderia ser considerada, relativa à interferência do conteúdo. Formas de uma realidade parcialmente escondida envolvem a criação de novidades, que poderiam estar ligadas à maior plasticidade das crianças para lidar com os confrontos proporcionados pelas situações de aprendizagem, evidenciando serem mais disponíveis para a mudança da estrutura de pensamento. Essa plasticidade aconteceria também nas relações interpessoais, permitindo-lhes maior objetividade na percepção.

Uma outra maneira de se entender por que a percepção funcionou mais em um conteúdo (formas de uma realidade parcialmente escondida) do que em outro (conservação de comprimento) poderia estar ligada às relações entre os aspectos funcionais e estruturais da cognição. As estruturas, no sistema piagetiano, podem ser consideradas como formas de organização da experiência e caracterizam, segundo Piaget (1985), o sujeito epistêmico, dotado de esquemas e estruturas estáveis, que the permitem "compreender" o real. A esse sistema presentativo fechado o autor acrescenta o sistema de procedimentos, em mobilidade contínua, que caracteriza o sujeito psicológico, aquele que busca "ter êxito" na satisfação de suas necessidades. O conteúdo formas de uma realidade parcialmente escondida envolveu, sobretudo, o sujeito psicológico e exigiu procedimentos mais imediatos e contextualizados. Também a percepção, considerada como fornecedora do conhecimento "imediato" da realidade exterior, foi tratada neste estudo como contextualizada, acontecendo na sala de aula. Ainda conforme Piaget, as realizações, o "saber fazer" (exigidos na prova dos possíveis) são mais precoces que as conceituações (exigidas na prova operatória).

Um outro ponto a considerar seria relativo à distinção feita por Piaget (1985) entre aspectos operativo e figurativo do conhecimento. O desenvolvimento da inteligência caracteriza-se pela constituição progressiva de um sistema de transformações, mas as realidades que o sujeito cognoscente procura conhecer englobam também estados, visto que cada transformação parte de um estado para outro. Os instrumentos e conhecimento denominados figurativos, nos quais a percepção se inclui, incidem sobre os estados ou traduzem os movimentos e transformações em termos de simples sucessão de estados. Nesse contexto, o conteúdo formas de uma realidade parcialmente escondida envolve predominantemente aspectos figurativos, de mais fácil apreensão, enquanto o conteúdo operatório envolve o sistema de transformações, de elaboração mais complexa cognitivamente, pois exigindo abstrações reflexivas.

$\mathrm{Na}$ realidade, analisando o conjunto dos dados, pode-se afirmar que alguns dos pontos abordados nesta pesquisa necessitam ainda de maiores esclarecimentos, sugerindo a necessidade de aprofundarem-se os estudos a respeito. Pesquisas com maior número de sujeitos, situações experimentais que esclareçam melhor a relação conteúdo da aprendizagem/percepção, incursões mais diretas no terreno educacional (ligação com aspectos afetivos e relacionais em sala de aula, com aprendizagem de conteúdos acadêmicos, etc.) poderiam ser relevantes para a complementação do presente estudo.

De toda maneira, este trabalho explorou um tema ainda controvertido na teoria piagetiana, ou seja, o das relações entre percepção e cognição em situações de aprendizagem, e especialmente no que se refere à percepção social. O próprio Piaget afirmou, em um estudo sobre as relações entre percepção e inteligência (Piaget., 1977), que o aprofundamento dessas questões poderia contribuir para um maior conhecimento, tanto da inteligência como das próprias percepções.

Permanecem questões como qual é exatamente o papel desempenhado pelo crescimento concomitante das estruturas intelectuais no desenvolvimento da atividade perceptiva? Qual a ação recíproca, se é que existe, da atividade perceptiva sobre a evolução das estruturas intelectuais? Com esses questionamentos, reitera-se a importância do tema que envolve percepção social e aprendizagem, pouco desenvolvido na teoria, mas talvez também, por isso mesmo, bastante promissor.

\section{Referências}

Bearison, D. J., Magzamen S. \& Filardo, E. M. (1986). Socio-cognitive conflict and cognitve growth in young children. Merril-Palmer Quartely, 32, (1), 51-72. 
Bijstra, J., Geert P. \& Jackson, S. (1989). Conservation and the appearance-reality distinction: what do children really know and what do they answer? British Journal of Developmental Pscychology, 7, 43-53.

Bustos, D. M. (1979). O Teste Sociométrico: fundamentos, técnicas e aplicações. São Paulo, SP: Brasiliense.

Cunha, C. C. (1999). Padrões de conduta de aprendizagem por conflito sociocognitivo em conteúdo operatório. (Tese de Doutorado). Campinas: Universidade Estadual de Campinas - Faculdade de Educação.

Emler, N. \& Glachan, M. (1988). Aprendizaje Social y Desarrolo Cognitivo. Em Mugny, G. \& Pérez, J. A. (Ed.). Psicologia social del desarrollo cognitivo. (pp. 95-118). Barcelona, Espanha: Antrophos Editorial del Hombre.

Fraysse, J. (1991). Effects of social insertion mode on performance and interaction assymetric dyads. European Journal of Psychology of Education, VI (1), 45-53.

Ganuza, F. B. (1996). Conflicto cognitivo y construcción de conocimientos. Em Ganuza, F. B. (Ed.). La construcción de conocimientos. (pp. 19-51). Bilbao: Servicio Editorial, Universidad del Pais Vasco.

Garcia, D. V. (1999). O conflito cognitivo como mediação entre maturação, gênero e aprendizagem. (Tese de Doutorado). Campinas: Universidade Estadual de Campinas Faculdade de Educação.

Krantz, M. (1982). Sociometric awareness, social participation and perceived popularity in preschool children. Child Development, 53, 376-379.

Maisonneuve, J. (1970). A sociometria e o estudo das relações preferenciais. Em Fraisse, P. \& Piaget, J. (pp. 225-280). Tratado de Psicologia Experimental. Rio de Janeiro, RJ: Forense.

Moreno, J. L. (1972). Fundamentos de la Sociometria. Buenos Aires: Paidós.

Mugny, G. \& Doise, W. (1983). La construcción social de la inteligencia. México: Trillas.

Mugny, G., Doise W. \& Giroud, J. C. (1975). Conflit de centrations et progrès cognitif, II: nouvelles illustrations expérimentales. Bulletin de Psychologie, 342, 979-985.

Mugny, G. Doise W. \& Perret-Clermont, A. N. (1975/76). Conflit de centrations et progrès cognitif. Bulletin de psychologie, 29, 199-204.

Nunes, L. D. (1998). Aprendizagem por conflito sociocognitivo e abertura de possiveis. (Dissertação de Mestrado em Psicologia Educacional). Campinas: Universidade
Estadual de Campinas - Faculdade de Educação.

Patterson, C. J., Kupersmidt, J. B. \& Griesler, P. C. (1990). Children's perceptions of self and of relationships with others as a function of sociometric status. Child Development, 61, 1335-1349.

Piaget, J. (1926). La representation du monde chez l'enfant. Paris: F. Alcan.

Piaget, J. (1973). Estudos sociológicos. Rio de Janeiro: Forense.

Piaget, J. (1977). O desenvolvimento do pensamento: equilibração das estruturas cognitivas. Lisboa: D. Quixote.

Piaget, J. (1985). O possivel e o necessário. Evolução dos possíveis na criança. Porto Alegre: Artes Médicas.

Piaget, J., Inhelder, B. \& Szeminska, A. (1948). La géometrie spontanée de l'enfant. Paris: Presses Universitaires de France.

Priel, B. \& Leshem, T. (1990). Self-perceptions of firstand second-grade children with learning disabilities. Journal of Learning Disabilities, 123(10), 637-642.

Russel, J. (1982). Cognitive conflict, transmission and justification conservation attainment through dyadic interaction. The Journal of Genetic Psychology, 140, 283297.

Sisto, F. F. (1993). Fundamentos para uma aprendizagem construtivista. Pró-Posições, 4(2), 38-52.

Sisto, F. F.(1997). Aprendizagem e Mudanças Cognitivas em Crianças. Petrópolis, R.J.: Vozes.

Taal, M. \& Oppenheimer, L. (1989). Socio-cognitive conflict and peer interaction: development of compensation. European Journal of Social Psychology, 19(1), 77-83.

Tagiuri, R. (1952). Relational analysis. Sociometry, 15, 91104.

Troncoso Guerrero, P. V. (1998). Interação Social: a dominância em situações de aprendizagem. (Dissertação de Mestrado). Campinas: Universidade Estadual de Campinas - Faculdade de Educação.

Tudge, J. (1989). When collaboration leads do regression: some negative consequences of socio-cognitive comflict. European Journal of Social Psychology, 19, 123-138.

Recebido em setembro de 2002

Reformulado em dezembro de 2002

Aprovado em março de 2003

Sobre a autora:

Fernanda Dias é psicóloga e pedagoga, mestre e doutora em Psicologia Educacional pela Universidade Estadual de Campinas, professora da Universidade Estadual de Londrina/PR. 